\title{
Analysis and Spectral Characteristics of a Spread-Spectrum Technique for Conducted EMI Suppression
}

\author{
K. K. Tse, Member, IEEE, Henry Shu-Hung Chung, Member, IEEE, S. Y. (Ron) Hui, Senior Member, IEEE, and \\ H. C. So, Member, IEEE
}

\begin{abstract}
Frequency modulation (FM) and random switching methods have been used for reducing conducted electromagnetic interference (EMI) in power converters. Limited theoretical studies and comparisons of these schemes, however, are available. In this paper, a detailed analysis and the spectral characteristics of a random carrier-frequency (RCF) technique for suppressing conducted EMI in an offline switched-mode power supply are presented. The analysis provides a theoretical platform for studying the characteristics of this random switching scheme. The level of randomness is defined for the RCF scheme and varied in the converter example so that its effects on the power spectra can be demonstrated. Theoretical predictions of the spectral characteristics of this scheme are confirmed with measurements. The RCF scheme has been compared with the standard constant-frequency pulsewidth modulation (PWM) scheme and the FM scheme. Comparisons of their spectral performance show that the RCF scheme has better conducted EMI suppression than the FM and standard PWM schemes.
\end{abstract}

Index Terms-AC-DC power conversion, pulsewidth modulation, random switching technique, switching circuit.

\section{INTRODUCTION}

$\mathbf{N}$ OWADAYS, switched-mode power supplies have to be designed not only to provide the required electrical functions, but also to meet international electromagnetic compatibility (EMC) standards. Inherently, switched-mode power supplies are electrically noisy. A typical configuration with the conducted electromagnetic interference (EMI) test setup is shown in Fig. 1 [1]. Two conflicting filter design constraints have to be considered. Firstly, in order to comply with the international EMI regulations, a differential and common-mode EMI filter is generally put in series with the line input. Secondly, for satisfying the safety requirements, the maximum values of the decoupling capacitors $C_{1}$ and $C_{2}$ (which are usually less than 0.01 $\mu \mathrm{F}$ and are connected between the supply lines and ground) are limited. These conflicting requirements make proper design of the noise filter not straightforward.

The switching device in a switched-mode power supply is the principle source of EMI. Decoupling capacitors $C_{1}, C_{2}$ and the parasitic capacitors $C_{p 1}-C_{p 5}$ form a closed loop. Part of the

Manuscript received August 25, 1998; revised July 7, 1999. This work was supported by the Hong Kong Research Council. Recommended by Associate Editor, J. Van Wyk.

The authors are with the Department of Electronic Engineering, City University of Hong Kong, Hong Kong (e-mail: eeshc@ @ityu.edu.hk).

Publisher Item Identifier S 0885-8993(00)02335-8. switch voltage will appear on $C_{1}$ and $C_{2}$. As the device's voltage consists of the fast switching edges, harmonics up to several megahertz may appear on $C_{1}$ and $C_{2}$, and hence on the supply network. The input current is generally pulsating and thus is rich in harmonic components. Most of the differential-mode noise can in principle be bypassed by the filter capacitors $C_{3}$ and $C_{4}$. Their capacitance values are dependent on the maximum peak of the harmonic components. However, the practical filtering effects of capacitors are not perfect. It is necessary to consider other alternatives so that the conducted EMI can be minimized.

The most effective way of suppressing conducted EMI is to act on the noise source. One obvious approach is to deal with the switching methods of the power device. Frequency modulation (FM) has been incorporated into the pulse-width-modulation (PWM) of the power converter [2] in order to reduce the input discrete harmonics. This approach is an important step forward in dealing with the conducted EMI issue because it offers a simple and yet effective method for modifying a standard PWM scheme to meet EMC standards. Recently, random switching technique has been recognized as an emerging technology for power converters [3]. Various random switching schemes, which are originated from statistical communication theory [11], have been reported for $\mathrm{dc} / \mathrm{ac}$ and $\mathrm{dc} / \mathrm{dc}$ power conversion [3]-[10]. The basic principle of introducing randomness into standard PWM scheme is to spread out the harmonic power so that no harmonic of significant magnitude exists. As a result, discrete harmonics are significantly reduced and the harmonic power is spread over the spectrum as 'noise' (continuous spectrum). Although some papers such as [12] have comparisons on PWM inverters, there is no reported comparison on these two approaches for offline switched-mode power supplies.

It has been pointed out in [10] that the continuous noise spectrum within the pass band of the converter's output low-pass filter could lead to noise-induced low-frequency voltage ripple in the converter's output. This undesirable feature may prohibit the use of random modulation in dc/dc converters, which require tight voltage regulation. In [10], this problem has been highlighted in the random pulse width modulation (RPWM) and random pulse position modulation (RPPM) schemes. For the RPWM method, the duty cycle of the switch is continuously changing in every switching cycle although the average duty cycle is theoretically equal to the nominal duty cycle. This duty cycle variation worsens the voltage ripple problem. In the RPPM scheme, there is also some possibility that two adjacent pulses of fixed duty cycle may appear together. Rigorous analyses of 


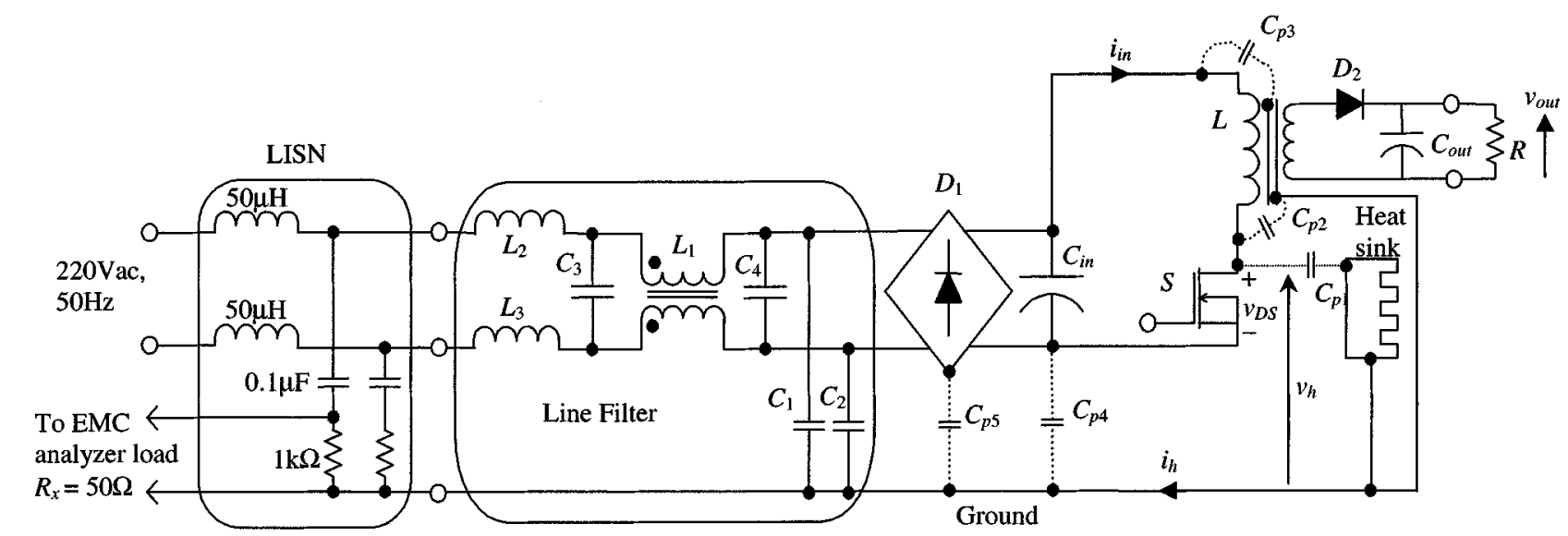

Fig. 1. Typical configuration of an offline switched-mode power supply with conducted EMI test setup.

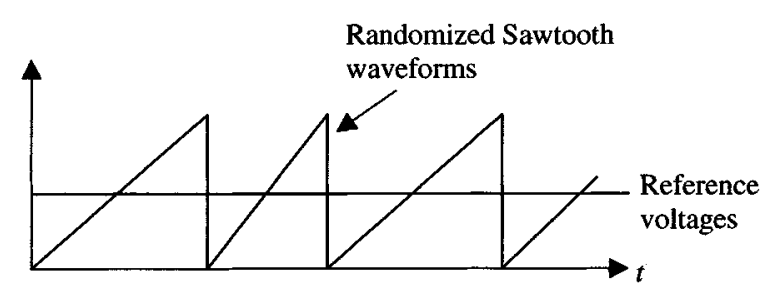

(a)

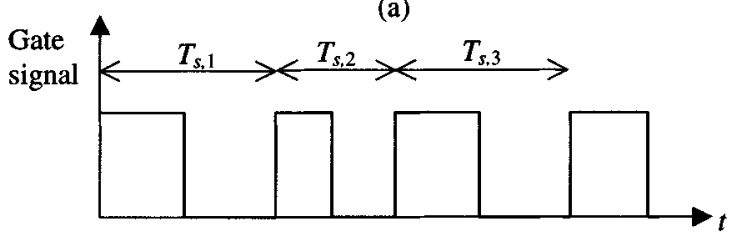

(b)

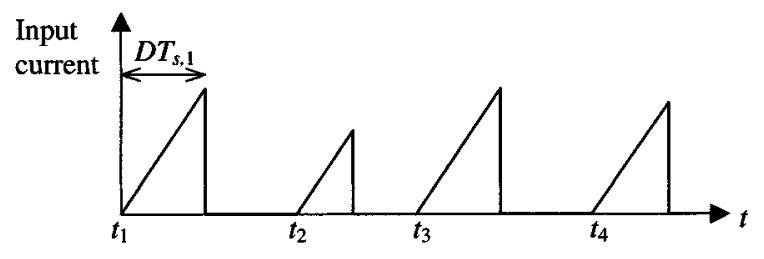

(c)

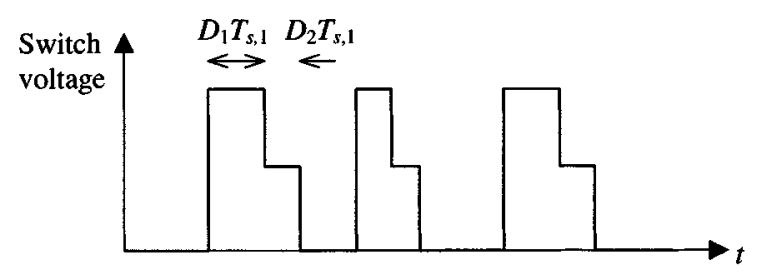

(d)

Fig. 2. Waveforms of flyback converter operating in discontinuous conduction mode with RCF scheme. (a) Sawtooth and reference voltage waveforms. (b) Gate signal. (c) Input current $i_{\text {in }}$. (d) Switch voltage $v_{\mathrm{DS}}$.

these two random schemes for $\mathrm{dc}-\mathrm{dc}$ converters have been presented in [13], [14].

In this paper, the random carrier frequency (RCF) scheme for dc-dc converter is examined and compared with the standard PWM scheme and the FM scheme. One attractive feature of the RCF scheme is that it inherently ensures constant
TABLE I

COMPONENT VALUES OF A PRACTICAL OFFLINE FLYBACK CONVERTER

\begin{tabular}{c|c}
\hline Converter & Line Filter \\
\hline$R=10 \Omega$ & $L_{1}=0.93 \mathrm{mH}$ \\
$L=1 \mathrm{mH}$ & $L_{2}=L_{3}=1 \mathrm{mH}$ \\
$C_{\text {in }}=220 \mu \mathrm{F}$ & $C_{1}=C_{2}=3.3 \mathrm{nF}$ \\
$C_{\text {out }}=470 \mu \mathrm{F}$ & $C_{3}=C_{4}=0.1 \mu \mathrm{F}$ \\
$D_{1}=1 \mathrm{~N} 4004 \mathrm{~s}$ & \\
$D_{2}=$ MUR460 & \\
$S=$ IRF840 & \\
Turns ratio $=5$ & \\
\hline
\end{tabular}

duty cycle operation in the dc-dc converter. The variation of the output voltage is not as significant as the RPWM and RPPM schemes and therefore allows simple feedback control design. At the same time, the power spectra of the converter input current and the switch voltage will spread over a wide frequency range so that no harmonic of significant magnitude exists. Effectively, the envelopes of the power spectra of the differential-mode and common-mode conducted EMI can in principle be reduced. These EMI aspects have been verified experimentally in [7]-[9]. However, the lack of theory of such scheme makes it difficult to decide how much randomness should be introduced. In this paper, a mathematical analysis of the above phenomena of the RCF method is given. The scope of this paper is not to answer all questions about the RCF scheme. Instead, it aims at providing a platform for understanding the spectral performance and the effect of the variation of the level of randomness. The model of an offline switched-mode power supply and mathematical derivations of the frequency spectra of the input current and the switch voltage waveform with and without RCF scheme are presented in Section II. Practical measurements of the conducted EMI of a 58-W, 220-V/24-V, 50-Hz offline flyback converter are given in Section III, together with analytical predictions. The results are compared with the standard constant switching frequency scheme and the FM [2] scheme. The conclusion follows in Section IV. 


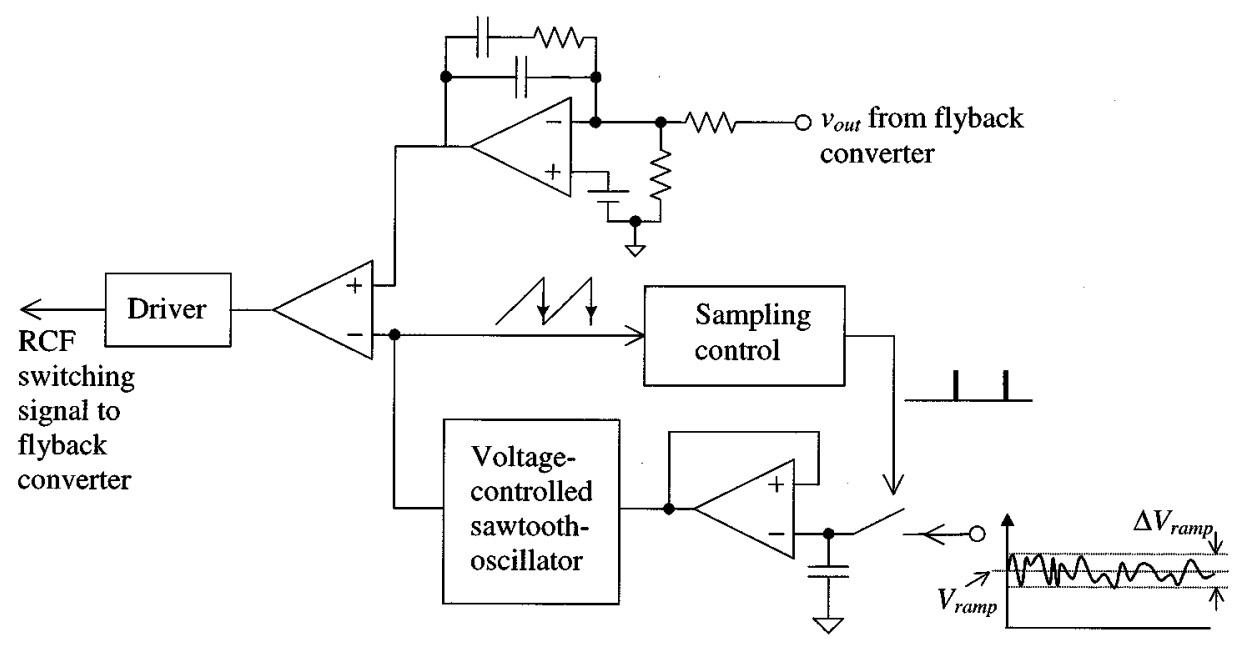

(a)

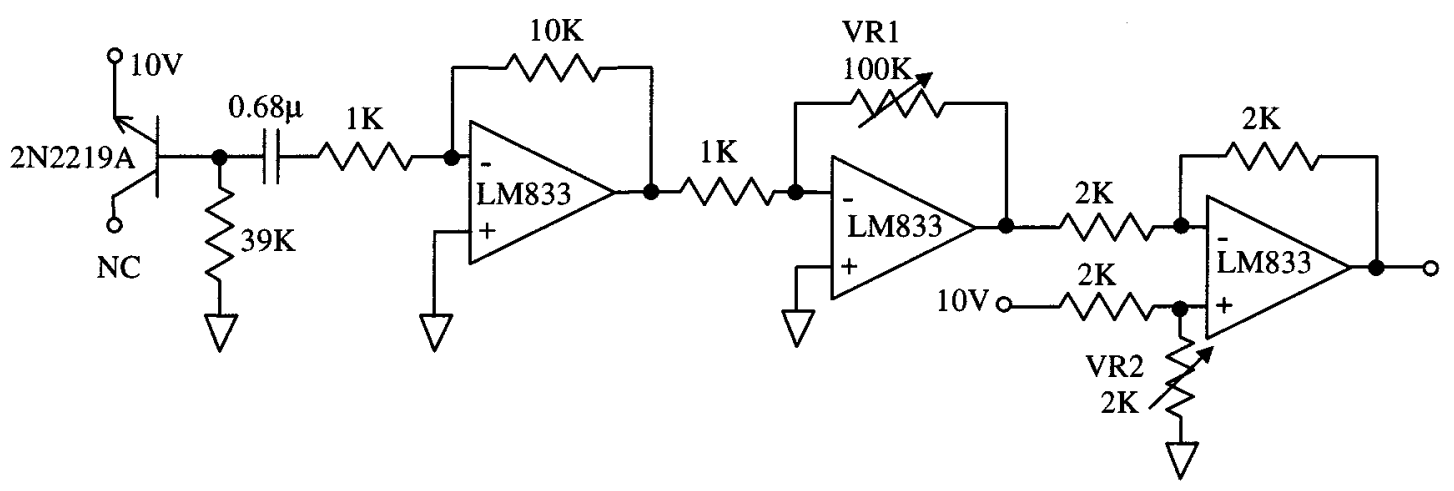

(b)

Fig. 3. Synthesis of RCF switching signal. (a) Feedback circuit. (b) Noise generator.

\section{Models AND MATHEMATiCAL DERIVATiOnS}

\section{A. Source of EMI}

As shown in Fig. 1, a standard line-impedance stabilization network (LISN) is connected between the power supply and the supply lines. As the supply source impedance is high, a common-mode harmonic current $i_{h}$ will flow through the ground plane, parasitic capacitance $C_{p 1}-C_{p 5}, C_{1}$, and $C_{2}$. If $C_{p}$ denotes the effective parasitic capacitance between the drain voltage of the switch $S$ (i.e., $v_{S}$ ) and the ground plane, the spectral magnitude of $i_{h}$ at frequency $f,\left|I_{h}(f)\right|$, can be obtained by

$$
\left|I_{h}(f)\right|=\left|V_{S}(f)\right| 2 \pi f C_{p}
$$

where $\left|V_{S}(f)\right|$ is the spectral magnitude of $v_{S}$. Thus, the spectral magnitude of the voltage across $C_{1}$ and $C_{2},\left|V_{h}(f)\right|$, can be approximated by

$$
\begin{aligned}
\left|V_{h}(f)\right| & =\left|I_{h}(f)\right| X_{C} \\
& =\frac{\left|I_{h}(f)\right|}{4 \pi f C}
\end{aligned}
$$

if $C_{1}=C_{2}=C$.
For the input side, since $L_{1}, L_{2}$, and $L_{3}$ form a potential divider with the simulated 50- $\Omega$ supply line resistance $R_{x}$ in the LISN, part of $v_{h}$ appears on $R_{x}$. Thus, in order to reduce the voltage across $R_{x}, L_{1}, L_{2}$, and $L_{3}$ can be increased. However, this will substantially decrease the voltage supplying to the converter circuit, due to the increase in the voltage drop across $L_{1}$, $L_{2}$, and $L_{3}$.

In practice, the value of $C_{p}$ and hence $i_{h}$ are slightly difficult to be determined. The above derivations are mainly for illustrating the major EMI source. In order to simplify the analysis, it is assumed that the switch voltage is not affected by the parasitic components. The validity of this assumption is ensured by the close agreement between the experimental measurements and the theoretical predictions.

In this study, the RCF scheme is applied to the PWM switching of the main switch $S$. Fig. 2(a) shows the randomized sawtooth waveform that is compared with a reference signal to generate the gate signal [Fig. 2(b)]. The duty cycle $D$ of $S$ is fixed in the respective cycle, although the switching period (stochastic variable) $T_{s}$ is varied. Thus, the differential-mode noise (due to the input current) and the common-mode noise (due to the switch voltage) can be spread over a certain frequency range. Spreading the harmonic power of the input current and the switch voltage signal is discussed separately in the following sub-sections. The mathematical derivations 


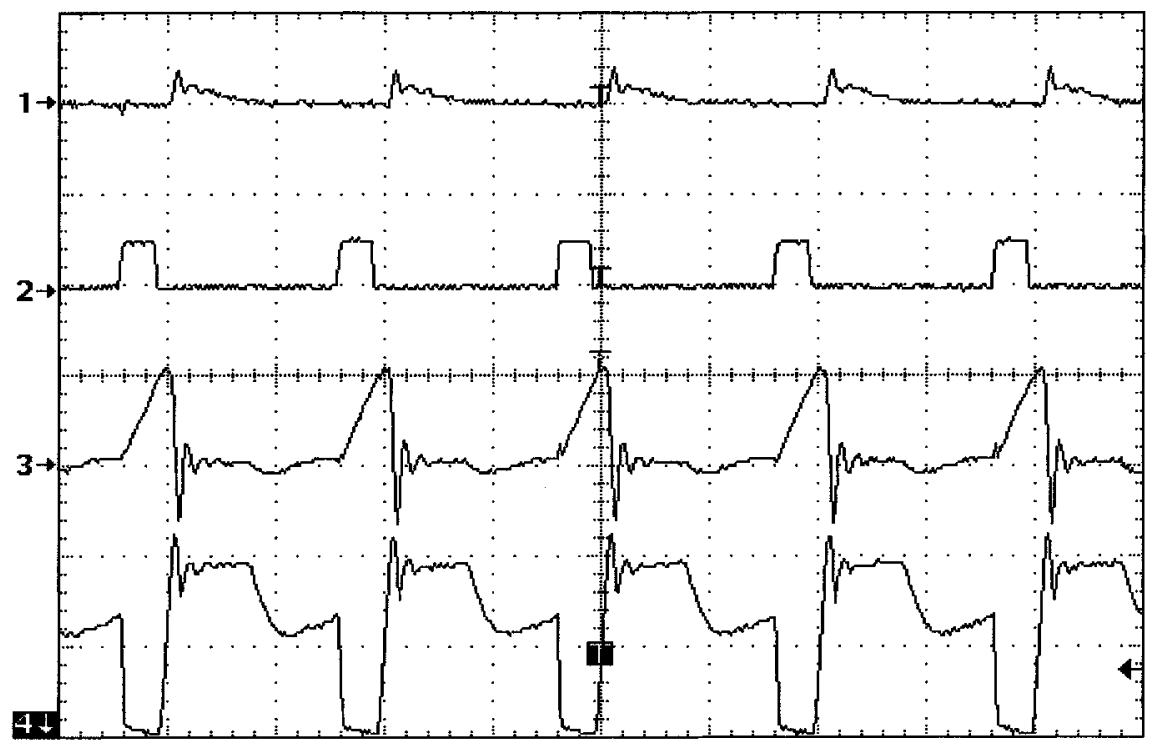

(a)

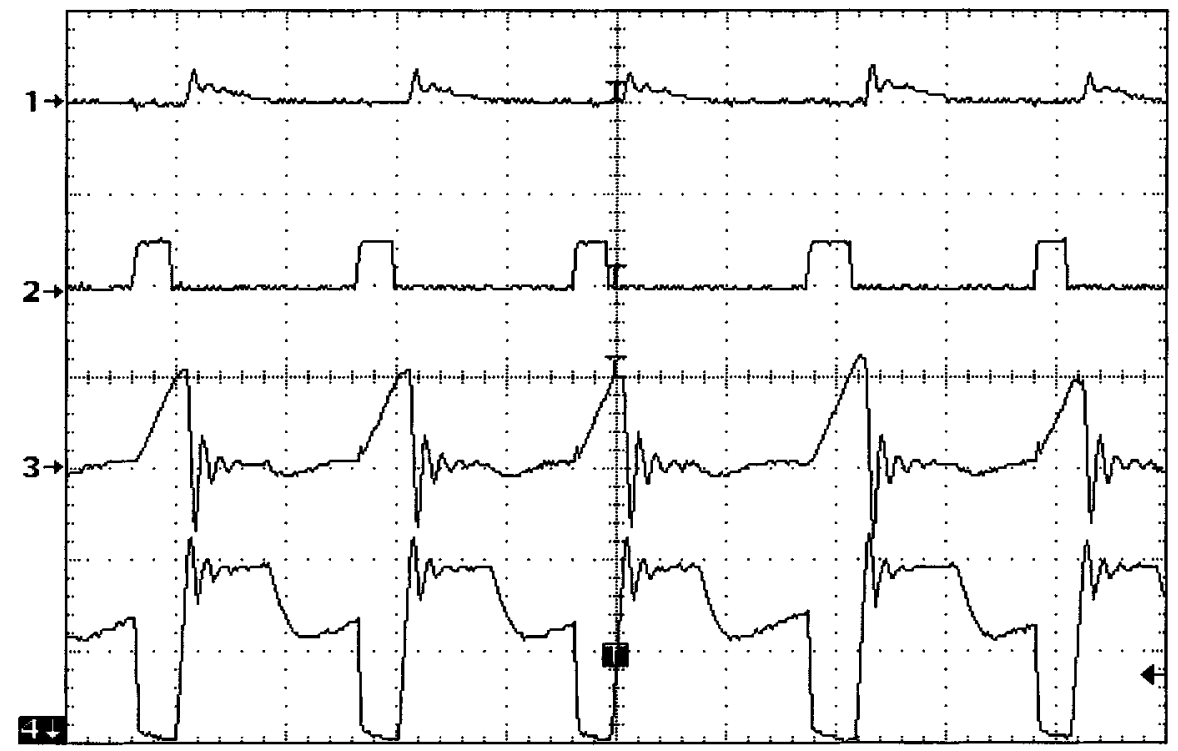

(b)

Fig. 4. Experimental waveforms of the converter. (a) $\Re=0$. (b) $\Re=0.2$. [Channel 1: Output voltage (1 V/div, with ac coupling). Channel 2: Gate signal (10 V/div). Channel 3: Input current (1 A/div). Channel 4: Switch voltage (200 V/div). Timebase: $10 \mu \mathrm{s} / \mathrm{div}$.]

are based on a flyback converter operating in discontinuous conduction mode (DCM), which is a commonly used configuration in many low-power applications. The attraction of the flyback converter is its single power conversion step, where the 'flyback' transformer provides the features of electrical isolation and as an energy storage medium.

\section{B. Spectral Characteristics of the Input Current Waveform}

Fig. 2(c) shows the waveform of the input current $i_{\text {in }}$ of the converter. For a generic switching cycle $k, T_{s}$ equals $T_{s, k}$. $i_{\text {in, }, k}(t)$ can be expressed as

$$
i_{\mathrm{in}, k}\left(t-t_{k}\right)= \begin{cases}\frac{V_{\mathrm{in}}}{L} t & \text { for } t_{k} \leq t \leq D T_{s, k} \\ 0 & \text { elsewhere }\end{cases}
$$

where $V_{\text {in }}$ is the input voltage and $L$ is the inductance of the flyback transformer. $T_{s}$ is a randomized switching period resulting from the RCF. The general expression of $i_{\text {in }}(t)$ is

$$
i_{\text {in }}(t)=\lim _{N \rightarrow \infty} \sum_{k=1}^{N} i_{\mathrm{in}, k}\left(t-t_{k}\right) .
$$

The auto-correlation of $i_{\text {in }}(t)$ is defined as

$$
R_{i_{\mathrm{in}}}(\tau)=E\left[\lim _{T_{o} \rightarrow \infty} \frac{1}{T_{o}} \int_{0}^{T_{o}} i_{\mathrm{in}}(t) i_{\mathrm{in}}(t+\tau) d t\right]
$$

where $E[\cdot]$ is the expected value of the quantity inside the bracket. $T_{o}$ is the observation interval, containing $N$ expected value of $T_{s}$. That is

$$
T_{o}=N E\left[T_{s}\right]
$$




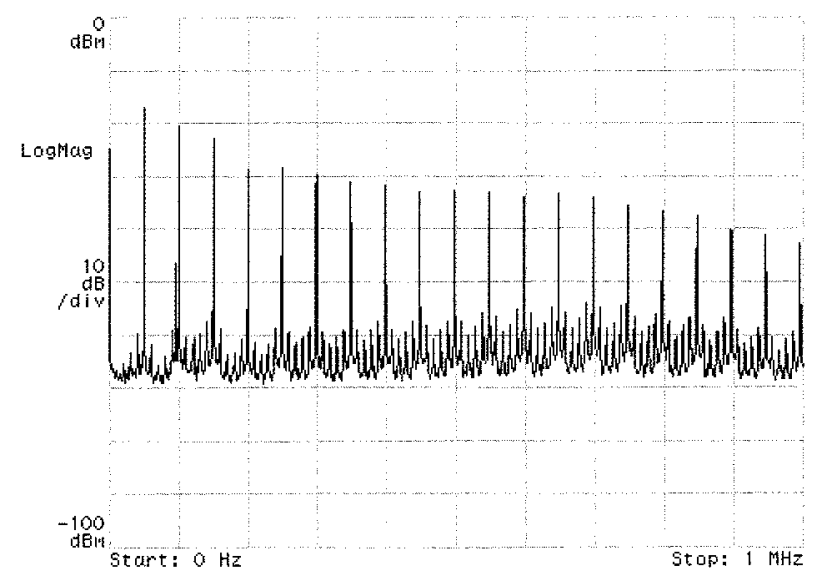

(a)

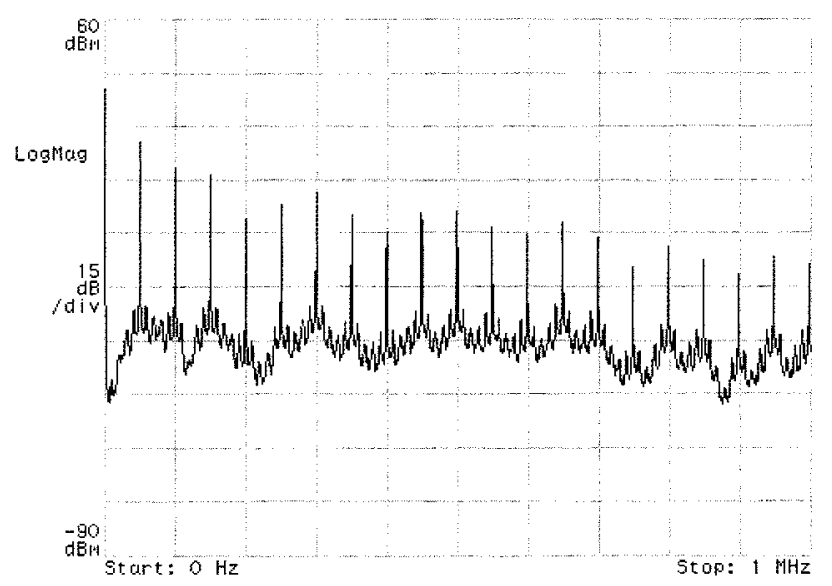

(c)

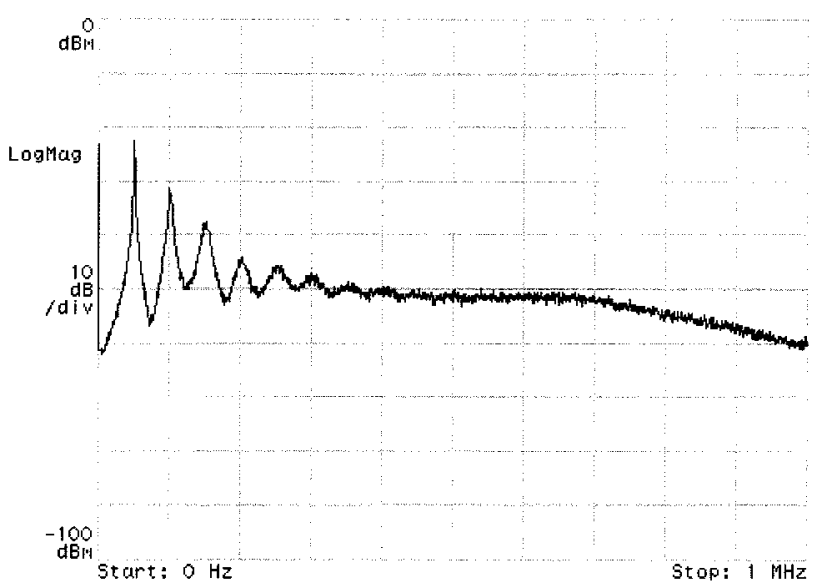

(b)

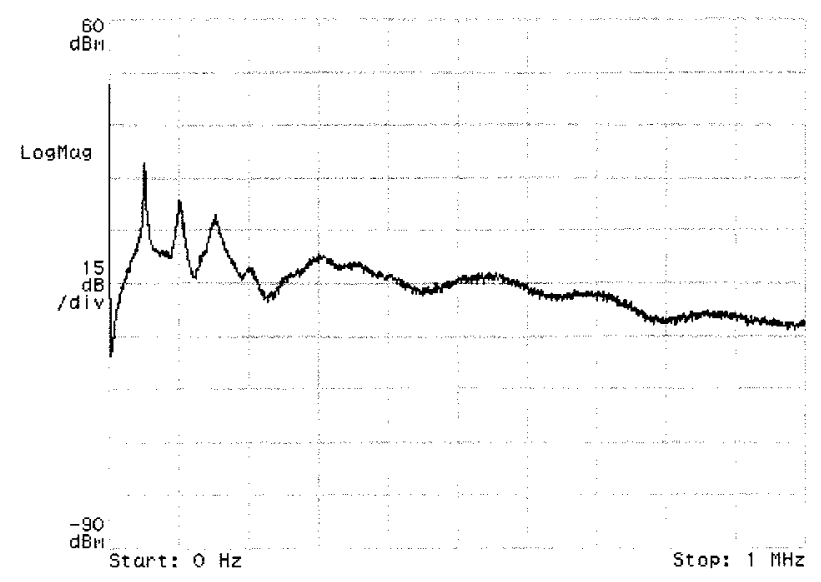

(d)

Fig. 5. Experimental power spectra. (a) Input current with $\Re=0$. (b) Input current with $\Re=0.2$. (c) Switch voltage with $\Re=0$. (d) Switch voltage with $\Re=0.2$.

By substituting (4) and (6) into (5)

$$
\begin{aligned}
R_{i_{\mathrm{in}}}(\tau)= & \lim _{N \rightarrow \infty} \frac{1}{N E\left[T_{s}\right]} \sum_{k=1}^{N} \sum_{l=1}^{N} \\
& \times E\left[\int_{0}^{\infty} i_{\mathrm{in}}\left(t-t_{k}\right) i_{\mathrm{in}}\left(t+\tau-t_{l}\right) d t\right] .
\end{aligned}
$$

If $I_{\mathrm{in}, k}(f)$ denotes the Fourier transform of $i_{\mathrm{in}, k}(t)$ and $\Gamma \mathrm{de}-$ notes the time integral in (7)

$$
\begin{aligned}
\Gamma= & \int_{0}^{\infty} i_{\mathbf{i n}}\left(t-t_{k}\right) i_{\mathbf{i n}}\left(t+\tau-t_{l}\right) d t \\
= & \int_{0}^{\infty}\left[\int_{-\infty}^{\infty} I_{\mathrm{in}, k}(f) e^{-j 2 \pi f t_{k}} e^{j 2 \pi f t} d f\right] \\
& \times\left[\int_{-\infty}^{\infty} I_{\mathrm{in}, l}\left(f^{\prime}\right) e^{j 2 \pi f^{\prime}\left(\tau-t_{l}\right)} e^{j 2 \pi f^{\prime} t} d f^{\prime}\right] d t \\
= & \int_{0}^{\infty} \int_{-\infty}^{\infty} \int_{-\infty}^{\infty} e^{j 2 \pi\left(f+f^{\prime}\right) t} d t I_{\mathrm{in}, k}(f) I_{\mathrm{in}, l}\left(f^{\prime}\right) \\
& \times e^{-j 2 \pi f t_{k}} e^{j 2 \pi f^{\prime}\left(\tau-t_{l}\right)} d f d f^{\prime} .
\end{aligned}
$$

Referring to [11]

$$
\int_{-\infty}^{\infty} e^{j 2 \pi\left(f+f^{\prime}\right) t} d t=\delta\left(f^{\prime}+f\right)
$$

Equation (8) can be expressed as

$$
\begin{aligned}
\Gamma= & \int_{0}^{\infty} \int_{-\infty}^{\infty} \delta\left(f^{\prime}+f\right) I_{\mathrm{in}, k}(f) I_{\mathrm{in}, l}\left(f^{\prime}\right) e^{-j 2 \pi f t_{k}} \\
& \times e^{j 2 \pi f^{\prime}\left(\tau-t_{l}\right)} d f d f^{\prime} \\
= & \int_{0}^{\infty} I_{\mathrm{in}, k}\left(-f^{\prime}\right) I_{\mathrm{in}, l}\left(f^{\prime}\right) e^{-j 2 \pi f^{\prime} \tau} e^{j 2 \pi f^{\prime}\left(t_{k}-t_{l}\right)} d f^{\prime} \\
= & \int_{0}^{\infty} I_{\mathrm{in}, k}^{*}(f) I_{\mathrm{in}, l}(f) e^{j 2 \pi f \tau} e^{j 2 \pi f\left(t_{k}-t_{l}\right)} d f
\end{aligned}
$$

Hence, the autocorrelation of $i_{\text {in }}$ can be expressed as

$$
\begin{aligned}
R_{i_{\text {in }}}(\tau)= & \lim _{N \rightarrow \infty} \frac{1}{N E\left[T_{s}\right]} \sum_{k=1}^{N} \sum_{l=1}^{N} \int_{0}^{\infty} \\
& \times E\left[I_{\mathrm{in}, l}(f) I_{\mathrm{in}, k}^{*}(f) e^{j 2 \pi f\left(t_{k}-t_{l}\right)}\right] e^{j 2 \pi f \tau} d f(11)
\end{aligned}
$$

Based on the Wiener-Khinchin theorem [11], the power spectrum of a signal $S(f)$ is the Fourier transform of its autocorrelation function $R(\tau)$. Conversely, the autocorrelation can be given by the inverse Fourier transform of the power spectrum. That is

$$
S(f)=\int_{-\infty}^{\infty} R(\tau) e^{-j 2 \pi f \tau} d \tau
$$




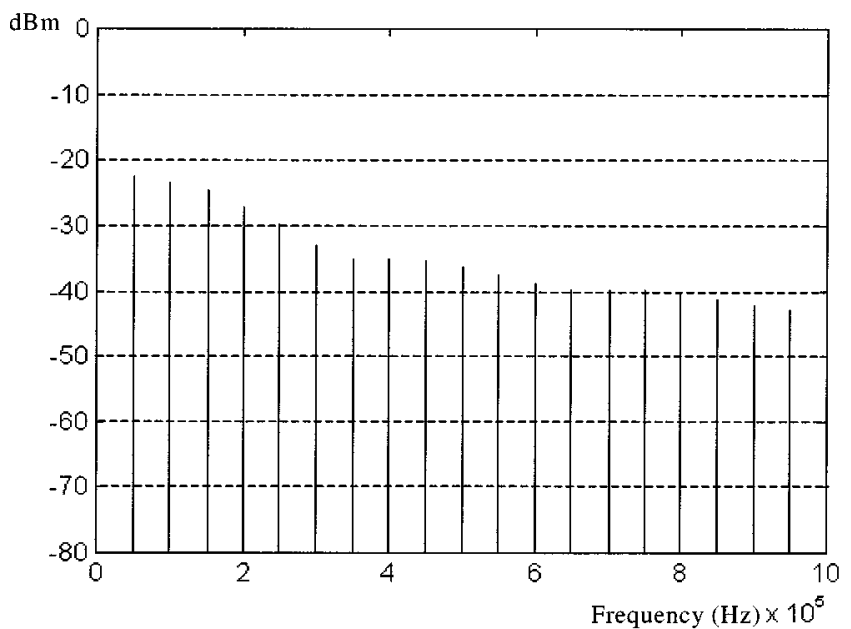

(a)

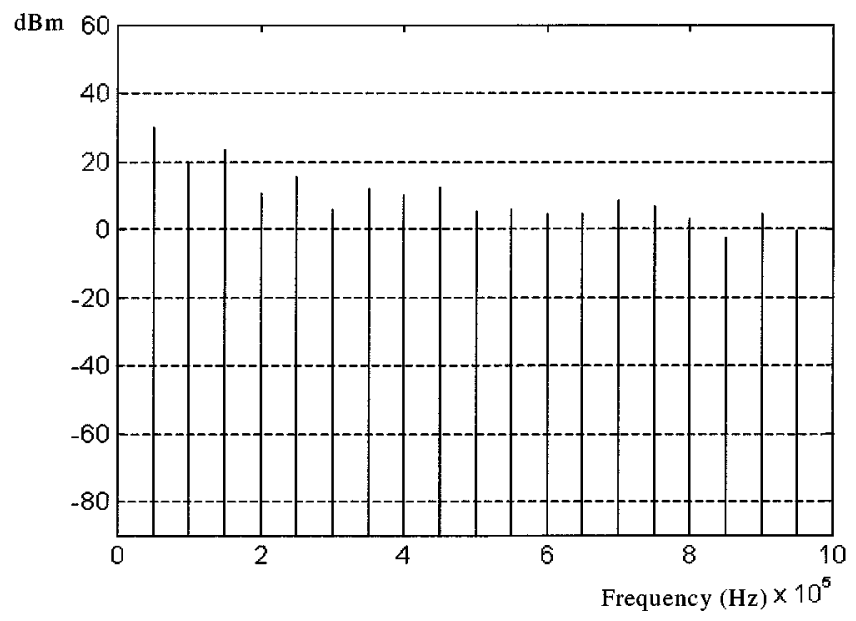

(c)

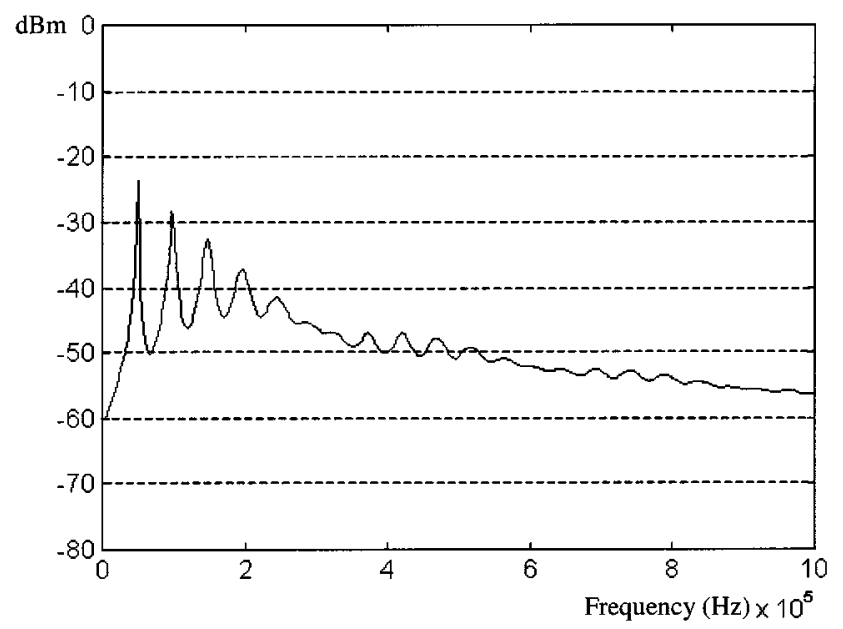

(b)

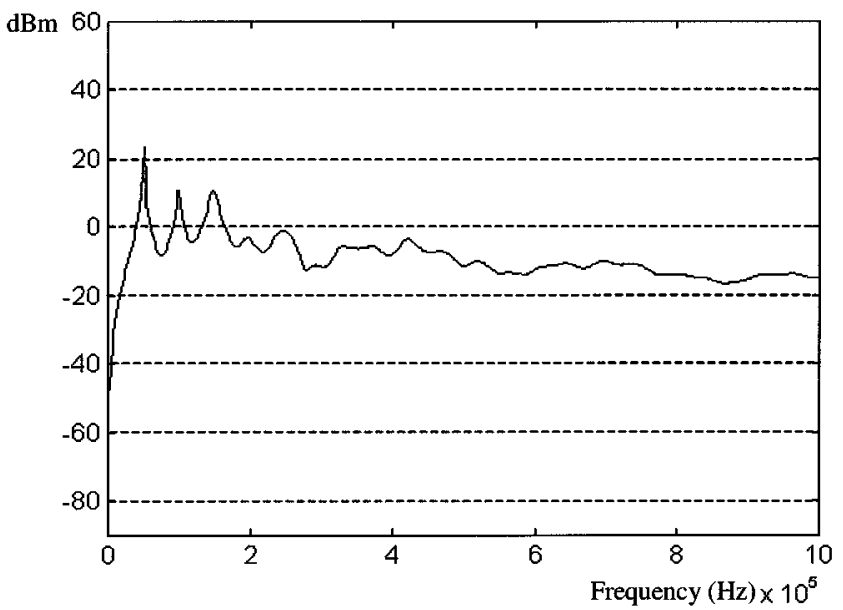

(d)

Fig. 6. Theoretical predictions of the power spectra. (a) Input current with $\Re=0$. (b) Input current with $\Re=0.2$. (c) Switch voltage with $\Re=0$. (d) Switch voltage with $\Re=0.2$.

and

$$
R(\tau)=\int_{-\infty}^{\infty} S(f) e^{j 2 \pi f \tau} d f
$$

By this relationship, the power spectrum of $i_{\text {in }}$ over the range of positive frequency, $S_{i_{\mathrm{in}}}(f)$, is easily observed from (11), i.e.,

$$
\begin{aligned}
S_{i_{\mathrm{in}}}(f)= & \lim _{N \rightarrow \infty} \frac{1}{N E\left[T_{s}\right]} \sum_{k=1}^{N} \sum_{l=1}^{N} \\
& \times E\left[I_{\mathrm{in}, l}(f) I_{\mathbf{i n}, k}^{*}(f) e^{j 2 \pi f\left(t_{k}-t_{l}\right)}\right] .
\end{aligned}
$$

The expected value of the summation in (14) forms a geometric series. Thus

$$
\begin{aligned}
& S_{i_{\mathrm{in}}}(f)= \frac{1}{E\left[T_{s}\right]}\left\{E\left[\left|I_{\mathrm{in}}(f)\right|^{2}\right]\right. \\
&\left.+2 \operatorname{Re}\left\{\frac{E\left[I_{\mathrm{in}}(f) e^{j 2 \pi f T_{s}}\right] E\left[I_{\mathrm{in}}^{*}(f)\right]}{1-E\left[e^{j 2 \pi f T_{s}}\right]}\right\}\right\} \\
& \text { for } f \neq 0 .
\end{aligned}
$$

Detailed proofs are shown in the appendix.
For $f=0$

$$
S_{i_{\mathrm{in}}}(0)=\frac{1}{\left\{E\left[T_{s}\right]\right\}^{2}}\left\{E\left[I_{\mathrm{in}}(0)\right]\right\}^{2} .
$$

It should be noted that (15) is a general expression for RCF switching schemes under consideration if $I_{\text {in }}(f)$ is substituted by the Fourier transform of a cycle of the considered signal. In Section II-C, the derivation of power spectrum of switch voltage is based on (15).

1) Standard Constant Switching Frequency (Standard) Scheme: If the converter operates with standard scheme, (14) is modified with

$$
I_{\mathrm{in}, l}(f)=I_{\mathrm{in}, k}(f)=I_{\mathrm{in}}(f)
$$

and

$$
t_{k+1}-t_{k}=T_{s} .
$$

where $T_{s}$ is a constant. The power spectrum can be shown to be

$$
S_{i_{\mathrm{in}}}=(f) \frac{1}{T_{s}}\left|I_{\mathrm{in}}(f)\right|^{2} \sum_{k=1}^{\infty} e^{j 2 \pi f k T_{s}} .
$$


Using the Poisson identity, the sum of exponential terms in (19) is defined as the sum of delta functions over the spectrum. That is

$$
\sum_{k=1}^{\infty} e^{j 2 \pi k f T_{s}}=f_{s} \sum_{k=1}^{\infty} \delta\left(f-k f_{s}\right)
$$

where $f_{s}=\left(1 / T_{s}\right)$.

Thus, the power spectrum of $i_{\text {in }}$ in the standard scheme is

$$
S_{i_{\mathrm{in}}}(f)=\frac{1}{T_{s}^{2}}\left|I_{\mathrm{in}}(f)\right|^{2} \sum_{k=1}^{\infty} \delta\left(f-k f_{s}\right)
$$

By (3), the Fourier transform of a cycle of $i_{\mathrm{in}}$ is

$$
\begin{aligned}
& I_{\mathrm{in}}(f) \\
& \quad=\int_{0}^{D T_{s}} \frac{V_{\mathrm{in}}}{L} t e^{-j 2 \pi f t} d t \\
& \quad=\frac{V_{\mathrm{in}}}{j 2 \pi f L} e^{-j \pi f D T_{s}}\left[\frac{\sin \left(\pi f D T_{s}\right)}{\pi f}-D T_{s} e^{-j \pi f D T_{s}}\right] .
\end{aligned}
$$

Thus, the squared absolute value is

$$
\begin{aligned}
\left|I_{\text {in }}(f)\right|^{2}= & \frac{2 V_{\text {in }}^{2}}{(2 \pi f)^{4} L^{2}}\left[1-\cos \left(2 \pi f D T_{s}\right)-2 \pi f D T_{s}\right. \\
& \left.\times \sin \left(2 \pi f D T_{s}\right)+\frac{\left(2 \pi f D T_{s}\right)^{2}}{2}\right] .
\end{aligned}
$$

For the standard scheme, $S_{i_{\text {in }}}(f)$ is determined by substituting (23) into (21).

2) RCF Scheme: Assume that the randomness of $T_{s}$ is subject to a probability density function $P\left(T_{s}\right)$, which has the uniform distribution with upper limit $T_{2}$ and lower limit $T_{1}$ (i.e., $\left.T_{s} \in\left[T_{1}, T_{2}\right]\right)$. Let $\Delta T_{s}$ denote the period deviation and $\Re$ denote the level of randomness on $T_{k}$

$$
\begin{aligned}
\Delta T_{s} & =T_{2}-T_{1} \\
\Re & =\frac{\Delta T_{s}}{E\left[T_{s}\right]} .
\end{aligned}
$$

Hence, the probability density function of $T_{s}$ is of the form

$$
P\left(T_{s}\right)= \begin{cases}\frac{1}{\Delta T_{s}}=\frac{1}{\Re E\left[T_{s}\right]}, & T_{1} \leq T_{s} \leq T_{2} \\ 0, & \text { otherwise }\end{cases}
$$

For a non-zero $\Re$, the expected values of the terms in (15) are found as follows:

$$
\begin{aligned}
E\left[T_{s}\right]= & \frac{T_{2}+T_{1}}{2} \\
E\left[\left|I_{\mathrm{in}}(f)\right|^{2}\right]= & \int_{T_{1}}^{T_{2}} P\left(T_{s}\right)\left|I_{\mathrm{in}}(f)\right|^{2} d T_{s} \\
= & \frac{2 V_{\mathrm{in}}^{2}}{(2 \pi f)^{4} L^{2} \Re E\left[T_{s}\right]}\left\{\Re E\left[T_{s}\right]\right. \\
& \quad-\frac{1}{\pi f D}\left[\sin \left(2 \pi f D T_{2}\right)-\sin \left(2 \pi f D T_{1}\right)\right] \\
& +T_{2} \cos \left(2 \pi f D T_{2}\right)-T_{1} \cos \left(2 \pi f D T_{1}\right) \\
& \left.+\frac{(2 \pi f D)^{2}}{6}\left(T_{2}^{3}-T_{1}^{3}\right)\right\} \cdots
\end{aligned}
$$

$$
\begin{aligned}
E[ & \left.I_{\mathrm{in}}(f) e^{j 2 \pi f T_{s}}\right] \\
= & \int_{T_{1}}^{T_{2}} P\left(T_{s}\right) I_{\mathrm{in}}(f) e^{j 2 \pi f T_{s}} d T_{s} \\
= & \frac{V_{\mathrm{in}}}{j(2 \pi f)^{3} L \Re E\left[T_{s}\right]}\left\{\left\{\frac{1}{1-D}\right.\right. \\
& \left.-\frac{D\left[1-j 2 \pi f(1-D) T_{2}\right]}{(1-D)^{2}}\right\} e^{j 2 \pi f(1-D) T_{2}} \\
& -\left\{\frac{1}{1-D}-\frac{D\left[1-j 2 \pi f(1-D) T_{1}\right]}{(1-D)^{2}}\right\} e^{j 2 \pi f(1-D) T_{1}} \\
& \left.-e^{j 2 \pi f T_{2}}+e^{j 2 \pi f T_{1}}\right\}
\end{aligned}
$$

$$
\begin{aligned}
E[ & \left.I_{\mathrm{in}}^{*}(f)\right] \\
= & \int_{T_{1}}^{T_{2}} P\left(T_{s}\right) I_{\mathrm{in}}^{*}(f) d T_{s} \\
= & \frac{V_{\mathrm{in}}}{j(2 \pi f)^{3} L D \Re E\left[T_{s}\right]}\left\{\left(2-j 2 \pi f D T_{2}\right) e^{j 2 \pi f D T_{2}}\right. \\
& \left.-\left(2-j 2 \pi f D T_{1}\right) e^{j 2 \pi f D T_{1}}-j 2 \pi f D \Re E\left[T_{s}\right]\right\} .
\end{aligned}
$$

By substituting (26)-(29) into (15), $S_{\dot{i}_{\text {in }}}(f)$ in the RCF scheme can be found. As will be shown in Section III, $S_{i_{\text {in }}}(f)$ will become close to a continuous spectrum as $\Re$ increases.

\section{Spectral Characteristics of the Switch Voltage Waveform}

The waveform of the drain-source voltage $v_{\text {DS }}$ of $S$ in Fig. 2(d) is expressed as

$$
v_{\mathrm{DS}}(t)= \begin{cases}0, & \text { for } 0 \leq t \leq D T_{k} \\ V_{\mathrm{in}}+n v_{\mathrm{out}}=V_{M}, & \text { for } D T_{k}<t \leq D_{1} T_{k} \\ V_{\mathrm{in}}, & \text { for } D_{1} T_{k}<t \leq T_{k}\end{cases}
$$

where $n$ is the turns ratio of the flyback transformer and $v_{\text {out }}$ is the output voltage, which is equal to

$$
v_{\mathrm{out}}=\sqrt{\frac{R T_{s}}{2 L}} D V_{\mathrm{in}}
$$

where $R$ is the output load resistance. $D$ is the expected value of the duty cycle in RCF scheme. It is the nominal value of the duty cycle in standard scheme.

$$
\begin{aligned}
D_{1} & =\frac{D V_{\text {in }}}{n v_{\text {out }}} \\
D_{2} & =1-D-D_{1} .
\end{aligned}
$$

Following the similar approach for $i_{\text {in }}$, the power spectrum $S_{v_{\mathrm{DS}}}(f)$ can be expressed as

$$
\begin{aligned}
& S_{v_{\mathrm{DS}}}(f)= \frac{1}{E\left[T_{s}\right]}\left\{E\left[\left|V_{\mathrm{DS}}(f)\right|^{2}\right]\right. \\
&\left.+2 \operatorname{Re}\left\{\frac{E\left[V_{\mathrm{DS}}(f) e^{\left.j 2 \pi f T_{s}\right] E\left[V_{\mathrm{DS}}^{*}(f)\right]}\right.}{1-E\left[e^{\left.j 2 \pi f T_{s}\right]}\right.}\right\}\right\} \\
& \text { for } f \neq 0 .
\end{aligned}
$$


For $f=0$ :

$$
S_{v_{\mathrm{DS}}}(0)=\frac{1}{\left\{E\left[T_{s}\right]\right\}^{2}}\left\{E\left[V_{\mathrm{DS}}(0)\right]\right\}^{2} .
$$

1) Standard Constant Switching Frequency (Standard) Scheme: For the standard scheme, $T_{s}$ is a constant and

$$
S_{v_{\mathrm{DS}}}(f)=\frac{1}{T_{s}^{2}}\left|V_{\mathrm{DS}}(f)\right|^{2} \sum_{k=1}^{\infty} \delta\left(f-k f_{s}\right) .
$$

The Fourier transform of a cycle of $v_{\mathrm{DS}}$ is

$$
\begin{aligned}
V_{\mathrm{DS}}(f)= & \int_{0}^{T} v_{\mathrm{DS}}(t) e^{-j 2 \pi f t} d t \\
= & V_{M} \frac{\sin \left(\pi f D_{1} T_{s}\right)}{\pi f} e^{-j \pi f D_{1} T_{s}} e^{-j 2 \pi f D T_{s}} \\
& +V_{\mathrm{in}} \frac{\sin \left(\pi f D_{2} T_{s}\right)}{\pi f} e^{-j \pi f D_{2} T_{s}} e^{-j \pi f\left(1-D_{2}\right) T_{s}}
\end{aligned}
$$

and hence, its squared absolute value is given as

$$
\begin{aligned}
\left|V_{\mathrm{DS}}(f)\right|^{2}= & \frac{2}{(2 \pi f)^{2}}\left\{V_{M}^{2}+V_{\mathrm{in}}^{2}-V_{M} V_{\mathrm{in}}\right. \\
& -V_{M} V_{\mathrm{in}} \cos \left[2 \pi f(1-D) T_{s}\right] \\
& -\left(V_{M}^{2}-V_{M} V_{\mathrm{in}}\right) \cos \left(2 \pi f D_{1} T_{s}\right) \\
& \left.-\left(V_{\mathrm{in}}^{2}-V_{M} V_{\mathrm{in}}\right) \cos \left(2 \pi f D_{2} T_{s}\right)\right\} .
\end{aligned}
$$

2) RCF Scheme: In order to calculate $S_{v_{\mathrm{DS}}}(f)$, (34) is used. With the same assumptions of randomness of $T_{s}$ in (25), the expected values of the terms in (34) can be expressed as follows,

$$
\begin{aligned}
& E\left\{\left|V_{\mathrm{DS}}(f)\right|^{2}\right\} \\
& =\int_{T_{1}}^{T_{2}} P\left(T_{s}\right)\left|V_{\mathrm{DS}}(f)\right|^{2} d T_{s} \\
& =\frac{2}{(2 \pi f)^{3 \Re} \Re\left[T_{s}\right]}\left\{\left(V_{M}^{2}+V_{\text {in }}^{2}-V_{M} V_{\text {in }}\right)(2 \pi f) \Re E\left[T_{s}\right]\right. \\
& -\frac{V_{M} V_{\mathrm{in}}}{1-D}\left\{\sin \left[2 \pi f(1-D) T_{2}\right]-\sin \left[2 \pi f(1-D) T_{1}\right]\right\} \\
& -\frac{\left(V_{M}^{2}-V_{M} V_{\text {in }}\right)}{D_{1}}\left[\sin \left(2 \pi f D_{1} T_{2}\right)-\sin \left(2 \pi f D_{1} T_{1}\right)\right] \\
& \left.-\frac{V_{\mathrm{in}}^{2}-V_{M} V_{\mathrm{in}}}{D_{2}}\left[\sin \left(2 \pi f D_{2} T_{2}\right)-\sin \left(2 \pi f D_{2} T_{1}\right)\right]\right\} \text {, }
\end{aligned}
$$

$$
\begin{aligned}
E & {\left[V_{\mathrm{DS}}(f) e^{j 2 \pi f T_{s}}\right] } \\
& =\int_{T_{1}}^{T_{2}} P\left(T_{s}\right) V_{\mathrm{DS}}(f) e^{j 2 \pi f T_{s}} d T_{s} \\
& =\frac{1}{(2 \pi f)^{2} \Re E\left[T_{s}\right]}\left\{j(2 \pi f) \Re E\left[T_{s}\right] V_{\mathrm{in}}\right. \\
& -\frac{V_{M}}{1-D}\left[e^{j 2 \pi f(1-D) T_{2}}-e^{j 2 \pi f(1-D) T_{1}}\right] \\
& \left.+\frac{V_{M}-V_{\mathrm{in}}}{D_{2}}\left(e^{j 2 \pi f D_{2} T_{2}}-e^{j 2 \pi f D_{2} T_{1}}\right)\right\},
\end{aligned}
$$

$$
\begin{aligned}
& E\left[V_{\mathrm{DS}}^{*}(f)\right] \\
& =\int_{T_{1}}^{T_{2}} P\left(T_{s}\right) V_{\mathrm{DS}}^{*}(f) d T_{s}
\end{aligned}
$$

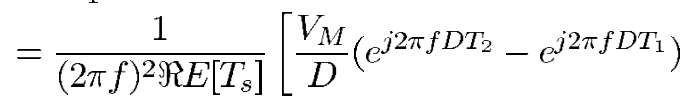

$$
\begin{aligned}
& +\frac{V_{\text {in }}-V_{M}}{D+D_{2}}\left(e^{j 2 \pi f\left(1-D_{2}\right) T_{2}}-e^{j 2 \pi f\left(1-D_{2}\right) T_{1}}\right) \\
& \left.-V_{\text {in }}\left(e^{j 2 \pi f T_{2}}-e^{j 2 \pi f T_{1}}\right)\right]
\end{aligned}
$$

Similar to the characteristics of the input current $S_{v_{\mathrm{DS}}}$ will be changed from a discrete harmonic spectrum to a continuous spectrum as the level of randomness in $T_{s}$ increases.

\section{EXPERIMENTAL VERIFICATIONS}

Fig. 3(a) shows the synthesis of RCF switching signal in the feedback network for the offline flyback converter shown in Fig. 1. The noise generator circuit is shown in Fig. 3(b). The transistor is used as a noise diode that gives constant noise signal strength over wide frequency band. The ripple of noise output $\Delta V_{\text {ramp }}$ and its DC value $V_{\text {ramp }}$ are adjustable by changing VR1 and VR2, respectively. The component values of the converter are tabulated in Table I. The output power of the converter is $58 \mathrm{~W}$. The output of the error amplifier is compared to a randomized sawtooth signal. A composite random signal, which contains a fixed dc signal $V_{\text {ramp }}$ and a noise with maximum amplitude $\Delta V_{\text {ramp }}$, generates the randomized sawtooth signal. The level of randomness $\Re$ in the RCF scheme can also be defined as

$$
\Re=\frac{\Delta V_{\text {ramp }}}{V_{\text {ramp }}}
$$

At the end of every switching cycle, the composite random signal is sampled and then fed to a voltage controlled oscillator (VCO) to generate the next sawtooth cycle. The nominal switching frequency is set at $50 \mathrm{kHz}$. $\Re=0$ corresponds to the standard PWM scheme and the switching frequency is 50 $\mathrm{kHz}$. When $\Re=0.2$, the switching frequency is uniformly randomized within the frequency range from $45 \mathrm{kHz}$ to $55 \mathrm{kHz}$. Fig. 4 shows the experimental voltage and current waveforms of the converter's main switch when $\Re=0$ and $\Re=0.2$, respectively. The gate signal is measured before the driver in Fig. 3(a). The output voltage under two cases is maintained at $24 \mathrm{~V}$ with ripple voltage of about $400 \mathrm{mV}$. In Fig. 4(a), the period of the input current pulses is fixed at $20 \mu$ s while the one in Fig. 4(b) is changed in every cycle. Although RCF can generate low-frequency noise within the pass band of the output filter, the output can be regulated to an acceptable level since the dynamics of the voltage output will be much slower than the crossover frequency of the feedback path. Hence the output voltage can be effectively stabilized as shown in Fig. 4.

Experimental measurements of the power spectra of $i_{\text {in }}$ [i.e., $\left.S_{\exp , i_{\text {in }}}(f)\right]$ and $v_{\mathrm{DS}}$ [i.e., $\left.S_{\exp , v_{\mathrm{DS}}}(f)\right]$ are shown in Fig. 5. The results were taken from a signal analyzer HP89410A with the use of the Hanning window, 4096 time samples, and a sampling rate of $2.5 \mathrm{MHz}$. 


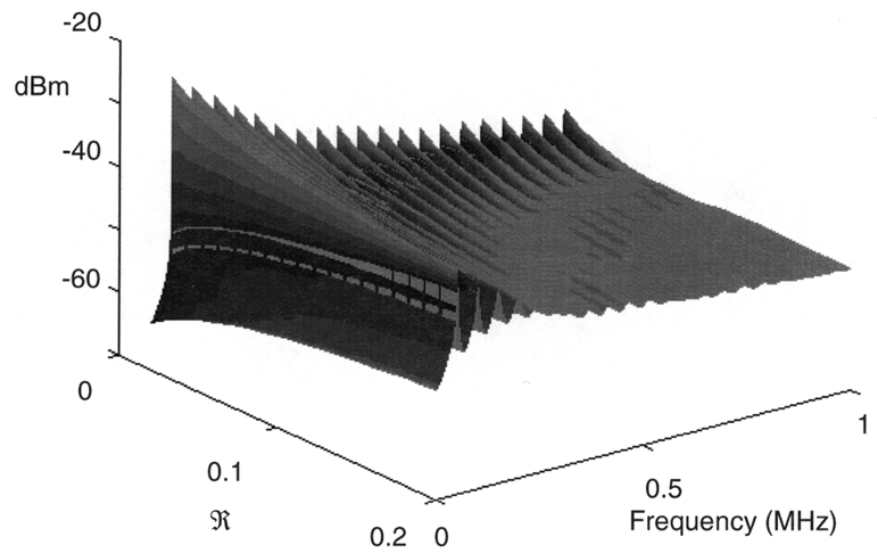

(a)

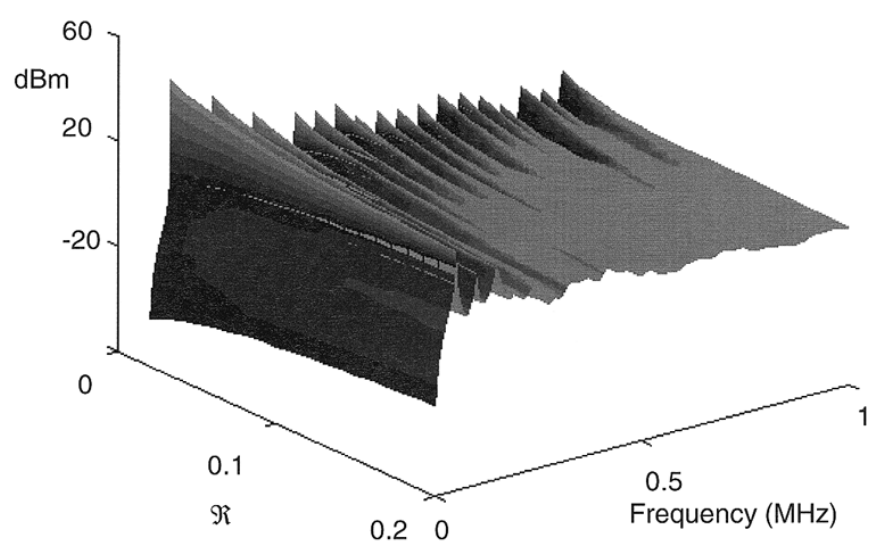

(b)

Fig. 7. Variation of power spectrum with $\Re$. (a) Input current. (b) Switch voltage.

It is important to note that the true power spectrum derived in Section II is based on infinite time-records of the RCF switching signal. However, as pointed out in [15], power spectra obtained from digital signal processing technique are strictly approximations because of the finite number of time records involved in the calculation. In order to make a better comparison of the theoretical power spectrum $S(f)$ and the experimental ones $S_{\exp }(f)$, a mathematical compensation for the analytical power spectrum $S^{\prime}(f)$ is performed by convoluting $S(f)$ with the window function $W$. That is

$$
\begin{aligned}
S^{\prime}(f) & =S\left(f_{0}\right) *\left|W\left(f_{0}\right)\right|^{2} \\
& =\frac{1}{2 \pi} \int_{-\infty}^{\infty} S(f)\left|W\left(f-f_{0}\right)\right|^{2} d f
\end{aligned}
$$

where $f_{0}$ is one of discrete frequency points given by FFT analysis, and the characteristic of the window function $W$ is subject to the following factor in FFT-based processing.

1) Type of windowing function.

2) Number of sampled time-record.

3) Sampling rate.

By considering the above factors, the modified analytical solution $S^{\prime}(f)$ is compared to the experimental $S_{\exp }(f)$ from the FFT-based spectrum analyzer, using the same parameters as HP89410A.

The analytical prediction of $S_{i_{\mathrm{in}}}^{\prime}(f)$ and $S_{v_{\mathrm{DS}}}^{\prime}(f)$ are shown in Fig. 6. All results are determined by taking the convolution

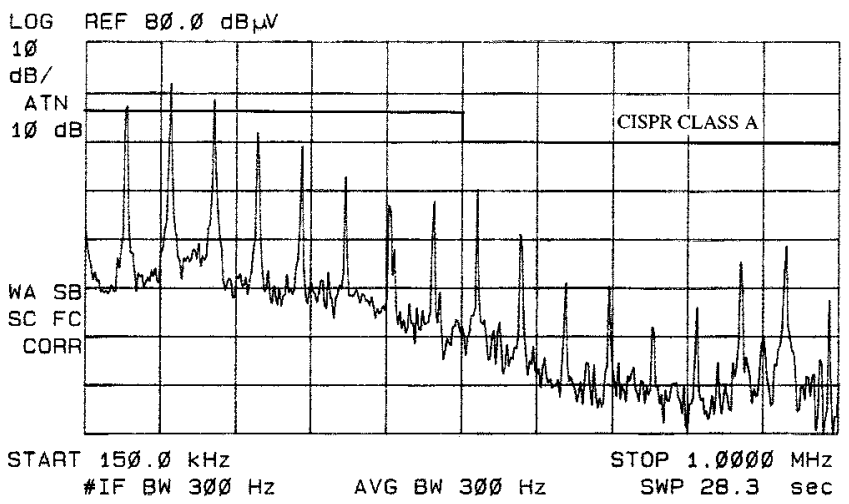

(a)

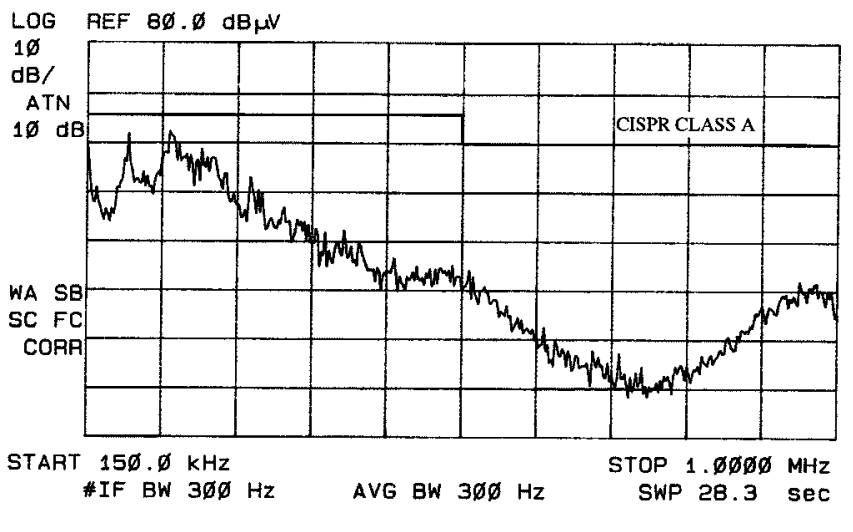

(b)

Fig. 8. Measured conducted emission. (a) $\Re=0$. (b) $\Re=0.2$.

(43) associated with the solutions of (15), (21), (34) and (36), respectively. Both analytical and experimental results are in close agreement. It can be observed that the RCF technique substantially reduces the discrete switching frequency harmonics. The peak harmonic power of the RCF scheme is much lower than that of the standard scheme. Using the theory developed, a threedimensional representation of the variations of the power spectrum with respect to the value of $\Re$ are generated and shown in Fig. 7. When $\Re$ equals zero, the operation is same as the standard PWM scheme. Discrete harmonics at multiples of the switching frequency can be observed. It is important to note that the power spectrum changes gradually from discrete harmonics spectrum to continuous noise spectrum with an increasing $\Re$. The overall envelope of the power spectrum also decreases as $\Re$ increases.

For the measurement of the conducted EMI of the converter, a LISN Farnell LSN30 and an EMC analyzer HP8591EM are used. Quasi-peak detector is used for all the testing. Fig. 8 shows the measured conducted emission under the above two cases and the limits of CISPR Publication 22 Class A devices. When $\Re=0$, some discrete harmonics exist and exceed the limit. With $\Re=0.2$, the harmonics are spread over. The spectrum is within the limit. Moreover, there is an approximately $10 \mathrm{~dB}$ reduction in the envelope of the spectrum with RCF. This can demonstrate the effectiveness of using RCF in suppression of EMI of the offline switched-mode power supply.

The RCF scheme is also compared with the FM scheme. Like the RCF scheme, the switching frequency of the FM scheme is allowed to vary within the frequency range from $45 \mathrm{kHz}$ to 55 


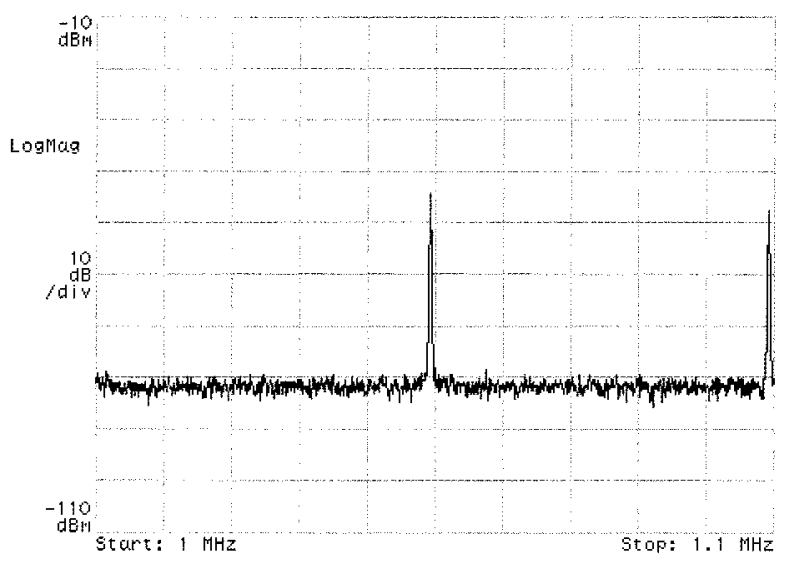

(a)

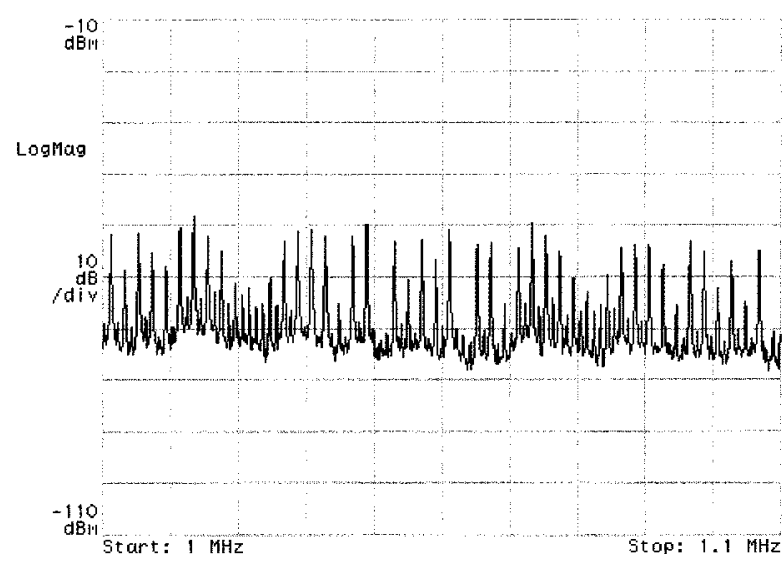

(b)

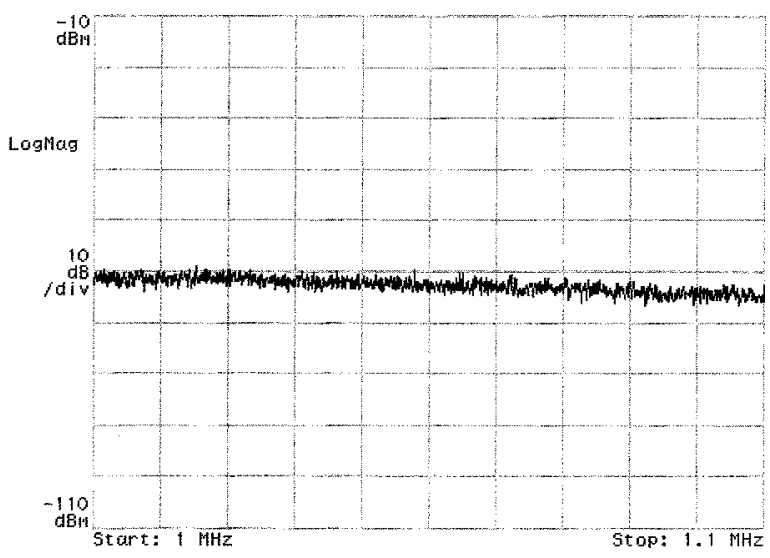

(c)

Fig. 9. Power spectra of the conducted emissions. (a) Standard PWM. (b) FM of $2 \mathrm{kHz}$ on standard PWM. (c) RCF scheme.

kHz. The FM scheme is modulated at $2 \mathrm{kHz}$. Fig. 9 shows a comparison of the conducted EMI when the converter is operated with

1) standard PWM [Fig. 9(a)];

2) FM of $2 \mathrm{kHz}$ on standard PWM [2] [Fig. 9(b)];

3) RCF scheme [Fig. 9(c)].

In order to investigate the details of the spectral characteristics, a frequency span of $1 \mathrm{MHz}-1.1 \mathrm{MHz}$ is studied for improving the frequency resolution. It can be seen that the FM scheme manages to reduce the discrete harmonic components to some extent when compared with the standard PWM scheme. The discrete harmonics are formed by the modulating frequency and the switching frequency [2]. Compared to the standard PWM, some discrete harmonics that do not exist in the standard PWM scheme are generated with the FM. However, the RCF scheme offers the best conducted EMI suppression among the three schemes under consideration.

\section{CONCLUSION}

An analysis on the random carrier frequency PWM method has been presented. The theory provides a mathematical platform for studying the spectral characteristic of this random PWM scheme. Spread spectrum switching technique disperses the discrete switching harmonics in standard PWM dc/dc converter as a wide-spectrum noise. It has the effect of reducing conducted EMI emissions as demonstrated in an experimental prototype. The RCF technique involves the randomization of the carrier frequency, but has the inherent feature of keeping duty cycle constant. The effects of the level of randomness on the degree of spectrum spreading are studied. Analytical prediction is verified with the practical measurements. The implementation here is simple and only involves a slight modification on existing circuits using standard PWM technique. In conclusion, the RCF scheme offers a simple solution to reduce conducted EMI in power converters. In terms of conducted EMI suppression, this study demonstrates that the RCF scheme is better than the FM scheme which has been incorporated into the PWM scheme of many commercial power converters.

\section{APPENDIX}

$S_{\Sigma}(f)$ denotes the double-summation of the expected term in (14); thus, (14) becomes

$$
\begin{aligned}
S_{i_{\mathrm{in}}}(f) & =\lim _{N \rightarrow \infty} \frac{1}{N E\left\{T_{s}\right\}} S_{\Sigma}(f) \\
S_{\Sigma}(f) & =\sum_{k=1}^{N} \sum_{l=1}^{N} E\left\{I_{\mathrm{in}, l}(f) I_{\mathrm{in}, k}^{*}(f) e^{j 2 \pi f\left(t_{k}-t_{l}\right)}\right\}
\end{aligned}
$$




$$
\begin{aligned}
S_{\Sigma}(f) & =N E\left[\left|I_{\mathrm{in}}(f)\right|^{2}\right] \\
& +\sum_{k=1}^{N} \sum_{\substack{l=1 \\
l \neq k}}^{N} E\left[I_{\mathrm{in}, l}(f) I_{\mathrm{in}, k}^{*}(f) e^{j 2 \pi f\left(t_{k}-t_{l}\right)}\right] .
\end{aligned}
$$

It should be noted that $I_{\mathrm{in}, l}(f)$ is dependent on $e^{j 2 \pi f\left(t_{k}-t_{l}\right)}$ if

$$
\left|t_{k}-t_{l}\right|=T_{s, l}
$$

and $I_{\mathrm{in}, k(f)}^{*}$ is dependent on $e^{j 2 \pi f\left(t_{k}-t_{l}\right)}$ if

$$
\left|t_{k}-t_{l}\right|=T_{s, k}
$$

Hence, (A.3) can be shown to be

$$
\begin{aligned}
S_{\Sigma}(f)= & N E\left[\left|I_{\mathrm{in}}(f)\right|^{2}\right]+2(N-1) \\
& \times \operatorname{Re}\left\{E\left[I_{\mathrm{in}}(f)\right] E\left[I_{\mathrm{in}}^{*}(f) e^{j 2 \pi f T_{s}}\right]\right\}+\Phi_{\Sigma}(f),
\end{aligned}
$$

where

$$
\begin{aligned}
\Phi_{\Sigma}(f)= & I_{\mathrm{in}, 1}^{*}(f) \sum_{l=3}^{N} I_{\mathrm{in}, l}(f) e^{j 2 \pi f\left(t_{1}-t_{l}\right)} \\
& +I_{\mathrm{in}, 2}^{*}(f) \sum_{\substack{l=4 \\
l \neq 1,2,3}}^{N} I_{\mathrm{in}, l}(f) e^{j 2 \pi f\left(t_{2}-t_{l}\right)} \\
& +I_{\mathrm{in}, 3}^{*}(f) \sum_{\substack{l=1 \\
l \neq 2,3,4}}^{N} I_{\mathrm{in}, l}(f) e^{j 2 \pi f\left(t_{3}-t_{l}\right)}+\cdots \\
& +I_{\mathrm{in}, N}^{*}(f) \sum_{l=1}^{N-2} I_{\mathrm{in}, l}(f) e^{j 2 \pi f\left(t_{N}-t_{l}\right)}
\end{aligned}
$$

Consider

$$
\phi=E\left[e^{j 2 \pi f T_{s}}\right]
$$

and

$$
E\left[e^{j 2 \pi f\left(t_{k}-t-l\right)}\right]= \begin{cases}{[\phi]^{k-l},} & \text { for } k>l \\ {\left[\phi^{*}\right]^{l-k},} & \text { for } l>k\end{cases}
$$

Thus, (A.7) becomes a real number as follow:

$$
\begin{aligned}
\Phi_{\Sigma}(f)= & 2 \operatorname{Re}\left\{E\left[I_{\mathrm{in}}^{*}(f)\right] E\left[I_{\mathrm{in}}(f) e^{j 2 \pi f T_{s}}\right]\right. \\
& \times\left(\phi+\phi+\phi^{2}+\phi+\phi^{2}+\phi^{3}+\cdots\right. \\
& \left.\left.+\phi+\phi^{2}+\phi^{3}+\phi^{4}+\cdots+\phi^{N-2}\right)\right\}
\end{aligned}
$$

Since $|\phi|<1$, (A.10) is simplified as

$$
\begin{aligned}
\Phi_{\Sigma}(f)= & 2 \operatorname{Re}\left\{E\left[I_{\mathrm{in}}^{*}(f)\right] E\left[I_{\mathrm{in}}(f) e^{j 2 \pi f T_{s}}\right]\right. \\
& \left.\times \frac{\phi}{1-\phi}\left[(N-1)-\frac{1-\phi^{N-1}}{1-\phi}\right]\right\} .
\end{aligned}
$$

By substituting (A.11) into (A.6):

$$
\begin{aligned}
S_{\Sigma}(f)= & N E\left[\left|I_{\mathrm{in}}(f)\right|^{2}\right]+2(N-1) \operatorname{Re}\left\{E\left[I_{\mathrm{in}}^{*}(f)\right]\right. \\
& \left.\times E\left[I_{\mathrm{in}}(f) e^{j 2 \pi f T_{s}}\right]\left(1+\frac{\phi}{1-\phi}\right)\right\} \\
& +2 \operatorname{Re}\left\{E\left[I_{\mathrm{in}}^{*}(f)\right] E\left[I_{\mathrm{in}}(f) e^{j 2 \pi f T_{s}}\right]\right. \\
& \left.\times \frac{\phi\left(1-\phi^{N-1}\right)}{(1-\phi)^{2}}\right\}
\end{aligned}
$$

Finally, (A.1) becomes

$$
\begin{aligned}
S_{i_{\mathrm{in}}}(f)= & \frac{1}{E\left\{T_{s}\right\}}\left(E\left\{\left|I_{\mathrm{in}}(f)\right|^{2}\right\}\right. \\
& \left.+2 \operatorname{Re}\left[\frac{E\left\{I_{\mathrm{in}}(f) e^{j 2 \pi f T_{s}}\right\} E\left\{I_{\mathrm{in}}^{*}(f)\right\}}{1-E\left\{e^{j 2 \pi f T_{s}}\right\}}\right]\right) .
\end{aligned}
$$

\section{REFERENCES}

[1] K. H. Billings, Ed., Switchmode Power Supply Handbook. New York: McGraw Hill, 1989.

[2] F. Lin and D. Y. Chen, "Reduction of power supply EMI emission by switching frequency modulation," in Proc. IEEE Power Electron. Spec. Conf., 1993, pp. 127-133.

[3] M. P. Kazmierkowski and F. Blaabjerg, "Impact of emerging technologies on PWM control of power electronic converters," IEEE Ind. Electron. Soc. Newsletter, pp. 9-13, Dec. 1995.

[4] A. M. Stankovic, G. C. Verghese, and D. J. Perreault, "Analysis and synthesis of randomised modulation schemes for power converters," IEEE Trans. Power Electron., vol. 10, no. 6, pp. 680-693, Nov. 1995.

[5] S. Y. R. Hui, I. Oppermann, and S. Sathiakumar, "Microprocessor based random PWM schemes for DC-AC power conversion," IEEE Trans. Power Electron., vol. 12, no. 2, pp. 253-260, Mar. 1997.

[6] T. Tanaka, T. Ninomiya, and K. Harada, "Random switching control in dc-dc converters," in Proc. IEEE Power Electron. Spec. Conf., 1989, pp. 500-507.

[7] D. S. Stone and B. Chambers, "The effect of carrier frequency modulation of PWM waveforms on conducted EMC problems in switched mode power supplies," EPE J., vol. 5, no. 3, pp. 32-37, Jan. 1996.

[8] — , "Effect of spread-spectrum modulation of switched mode power converter PWM carrier frequencies on conducted EMI," Electron. Lett., vol. 31, no. 10, pp. 769-770, May 1995.

[9] — "Easing problems in switched mode power converters by random modulation of the PWM carrier frequency," in Proc. Appl. Power Electron. Spec. Conf., 1996, pp. 327-332.

[10] Y. Shrivastava, S. Y. R. Hui, S. Sathiakumar, H. Chung, and K. K. Tse, "Effects of continuous noise in randomised switching dc-dc converters," Electron. Lett., vol. 33, no. 11, pp. 919-921, May 1997.

[11] D. Middleton, An Introduction to Statistical Communication Theory. New York: McGraw-Hill, 1988.

[12] J. T. Boys, "Theoretical spectra for narrow-band random PWM waveforms," Proc. Inst. Elect. Eng. B, vol. 140, no. 6, Nov. 1993.

[13] Y. Shrivastava, S. Y. R. Hui, S. Sathiakumar, H. Chung, and K. K. Tse, "Harmonic analysis of non-deterministic switching methods for dc-dc power converters," IEEE Trans. Circuits Syst.-Part I. , to be published.

[14] Y. Shrivastava, S. Y. R. Hui, S. Sathiakumar, K. K. Tse, and H. Chung, "A comparison of deterministic and non-deterministic switching methods for dc-dc converters," IEEE Trans. Power Electron., vol. 13, pp. 1046-1055, Nov. 1998.

[15] M. M. Bech, J. K. Pedersen, F. Blaabjerg, and A. M. Trzynadlowski, "A methodology for true comparison of analytical and measured frequency domain spectra in random PWM converters," in Proc. IEEE Power Electron. Spec. Conf., 1998, pp. 36-43. 


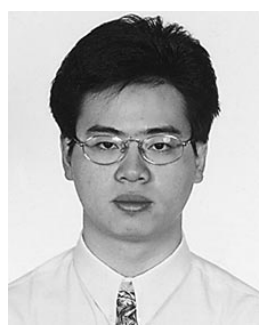

K. K. Tse ( $\left.\mathrm{S}^{\prime} 95\right)$ received the B.Eng. degree in electrical engineering (with honors) from the Hong Kong Polytechnic University, Hong Kong, in 1995 and the Ph.D. degree from the Department of Electronic Engineering, City University of Hong Kong, Hong Kong, in 2000.

He is a Research Fellow with the Department of Electronic Engineering, City University of Hong Kong. He has authored over eighteen technical papers in his research interests, which include computer-aided simulation technique, numerical modeling methods, EMI reduction, and random switching scheme for dc-dc converters.

Dr. Tse received First Prize in the 1998 IEEE Postgraduate Student Paper Contest, IEEE Hong Kong Section, and third prize in 1998 IEEE Region 10 Postgraduate student paper contest.

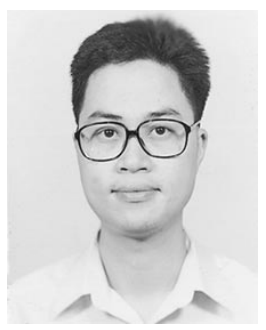

Henry Shu-Hung Chung (S'92-M'95) received the B.Eng. degree (with first class honors) in electrical engineering and the Ph.D. degree from The Hong Kong Polytechnic University, Hong Kong, in 1991 and 1994, respectively.

Since 1995, he has been with the City University of Hong Kong, where he is currently an Associate Professor in the Department of Electronic Engineering. His research interests include time- and frequency-domain analysis of power electronic circuits, switched-capacitor-based converters, random-switching techniques, digital audio amplifiers, fuzzy-logic control, and soft-switching converters. He has authored over 105 technical papers including over 47 journal papers.

Dr. Chung received the China Light and Power Prize and was awarded the Scholarship and Fellowship of the Sir Edward Youde Memorial Fund, in 1991 and 1993, respectively. He is currently Chairman of the Council of the Sir Edward Youde Scholar's Association and IEEE student branch counselor. $\mathrm{He}$ was track chair of the technical committee on power electronics circuits and power systems of IEEE Circuits and Systems Society in 1997-1998. He is currently an Associate Editor of the IEEE TRANSACTIONS ON C IRCUITS AND SYSTEMS-PART I. He has also been listed in Marquis Who's Who in the World.

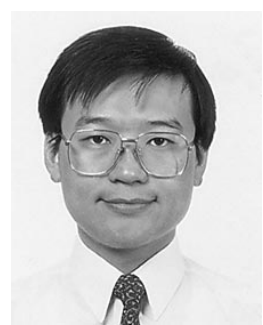

S. Y. (Ron) Hui (SM'94) was born in Hong Kong in 1961. He received the B.Sc. degree (with honors from the University of Birmingham, U.K. in 1984, and the D.I.C. and Ph.D. degrees from the Imperial College of Science, Technology, and Medicine, London, U.K., in 1987.

He was a Lecturer in power electronics at the University of Nottingham, U.K. from 1987 to 1990 . In 1990, he went to Australia and took up a lectureship at the University of Technology, Sydney, Australia, where he became a Senior Lecturer in 1991. Later, he joined the University of Sydney and was promoted to Reader of Electrical Engineering and Director of Power Electronics and the Drives Research Group in 1996. He is now a Chair Professor of Electronic Engineering at the City University of Hong Kong. He has published over 130 technical papers, including over 70 refereed journal publications. His research interests include all aspects of power electronics.

Dr. Hui is a Fellow of the IEE, the IEAust and the HKIE. He is an Associate Editor of the IEEE TRANSACTIONS ON POWER ELECTRONICS.

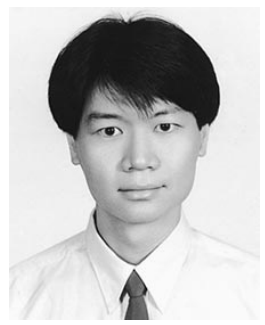

H. C. So (M'95) was born in Hong Kong in 1968. He received the B.Eng. degree in electronic engineering from the City Polytechnic of Hong Kong, Hong Kong, in 1990 and the Ph.D. degree in electronic engineering from the Chinese University of Hong Kong, Hong Kong.

From 1990 to 1991, he was an Electronic Engineer with the Research and Development Division, Everex Systems Engineering, Ltd., Hong Kong. From 1995 to 1996, he worked as a Post-Doctoral Fellow at the Chinese University of Hong Kong. He is currently a Research Assistant Professor in the Department of Electronic Engineering, City University of Hong Kong. His research interests include adaptive signal processing, detection and estimation, source localization, and wavelet transform. 